gram to strengthen multi-disciplinary disaster response capabilities in low-income countries by such collaborative efforts across civilian and military institutions that may cross national boundaries.

Methods: India is a disaster-prone, developing country with no formal national guidance for training in disaster preparedness for health sector. A massive earthquake in 2001 in Gujarat underlined the need for structured training in disaster preparedness for hospitals, prehospital personnel, and other health professionals. The Academy of Traumatology (India) in collaboration with the United Kingdom Defence Medical Services designed an Indiaspecific training program for disaster preparedness: the NDPCH. This is partly based on the MIMMS course, which is available in civilian and military modules in many developed countries. The NDPCH is a combination of the one-day MIMMS program that is directed to prehospital staff and a one-day course tailored to hospital-based staff. This two-day intensive program involves lectures, tabletop exercises, practical skills training (radio procedure, triage), and directed discussion. An assessment of practical skills takes place at the end of each day. To date, $>250$ professionals have been trained, and the course has become a national standard for training in disaster preparedness. The NDPCH initiative has led to increased awareness about disaster preparedness in India. The principles taught in the course were first put into practice in 2002 following a terrorist attack on the Akshardham Temple in Gandhinagar, Gujarat. The program also has stimulated the development of emergency medical services (EMS) in many cities. "Training-of-trainers," courses now are offered jointly by military and civilian faculty.

Conclusions: This civilian-military collaboration has proved a unique joint effort, which successfully has augmented the disaster response capability of the civilian health sector in a developing country through a tailormade training program based on international standards. This initiative has crossed national boundaries to promote cooperation between Europe and Asia. This course can serve as a model for military institutions with advanced capabilities in developed countries to collaborate with and support the disaster response capabilities in developing countries in order to strengthen the global efforts to reduce the burden of disasters.

Keywords: civilian-military; developing countries; Gujarat; India; MIMMS; National Disaster Preparedness Course for Hospitals (NDPCH), training

Prebosp Disast Med 2005;20(2):s84-s85

\section{Civilian Cooperation in Disaster Medicine}

\section{Klein; ${ }^{1}$ J. Storek ${ }^{2}$}

1. Charles University Prague, Czech Republic

2. Institute for Postgraduate Education, Prague, Czech Republic

Since the end of the Cold War and the fall of the Berlin Wall, rapid changes have taken place in the security function. The threat of military, global confrontation has been reduced greatly, and accordingly, the role of the military has changed. Political authorities are more likely to request military intervention oriented towards humanitarian assistance and disaster relief. Traditionally, military medical services have paved the way in these tasks. Well-trained personnel, organized rescue teams, and groups of specialists with appropriate technology must now be prepared to react at any time. These are vital factors in the immediate response to any disaster situation. In long-term-assistance operations, the military has the capability to help in the restoration of previously existing sanitary or healthcare systems. The whole area of civil-military relations and cooperation is defined and described in many NATO documents under the common term "CIMIC".

For example the MC 411/1 (NATO Military Policy on Civil-Military Cooperation) defines the wide and differing nature of relations between military and civil authorities, organizations, and agencies, and also the parameters of those relations according to the type of activities undertaken. In the Czech Republic, a specific law exactly defines the roles of individual bodies-civilian and military-involved in the response to any extraordinary or mass-casualty situations. An Integrated Rescue System (IRS) was installed to cover the whole country and recently has proved its effectiveness. During the flood disaster in August 2002 (the highest water level in the Czech Republic/Central European region for 500 years), military-civilian cooperation worked well within the framework of previously prepared legislation.

Keywords: CIMIC; Czech Republic; disaster medicine; mass-casualty; military-civilian cooperation; NATO

Prehosp Disast Med 2005;20(2):s85

Internalization of Medical Protocols during Medical Responses to Conventional and Unconventional Mass-Casualty Incidents

\section{O. Benin-Goren; ${ }^{1}$ A. Blumenfeld ${ }^{2}$}

1. Tel Aviv Sourasky Medical Center, Israel

2. Medical Corps, Israeli Defense Forces, Israel

Medical care in mass-casualty incidents (MCIs) is significantly different from medical and trauma care on a daily basis. MCIs due to terrorism creates challenges to medical response in the field and in hospitals. Medical personnel at all levels, on-site and in hospitals, share the objective to reduce mortality and morbidity caused by MCIs. Due to the security situation in Israel, injuries, such as those resulting from blasts or gunshots formerly identified with the battle zone, strike the civilian population.

Army Medical Corps and civil medical services cooperated and shared forces in order to research and establish national protocols for medical care due to MCIs. Civilian-military collaboration is part of emergency preparedness in Israel at several levels; as part of national committees at the level of policymakers, along with other civil members and ministers from the Ministry of Health $(\mathrm{MOH})$, in the field level Medical Corp (MC) are taking part of training program related to conventional $\mathrm{MCI}$ as well as nuclear, biological and chemical (NBC) preparedness.

As part of readiness for $\mathrm{MCI}$, the $\mathrm{MC}$ in Israel have the highest capability to provide four essential elements: (1) search and rescue; (2) triage and initial stabilization; (3) medical care and evacuation with vehicle evacuation that may be 
based on exchange of military (armored) and.civilian ambulances; and (4) helicopter evacuation that is exclusively military. Civilian-military collaboration creates a combination of joined forces, fast response, and standards of work in the field and in hospitals. All can help reduce high mortality and morbidity due to MCIs. This work will present the areas of civilian-military collaboration in Israel and the advantages of this collaboration, based on past experience.

Keywords: civilian-military collaboration; Israel; mass-casualty incident; response

Prehosp Disast Med 2005;20(2):s85-s86

\section{Military-Civil Cooperation Issues in Disaster Medicine in Acts of Terrorism}

S. Goncharov

All-Russian Centre for Disaster Medicine, Russia

The concept of military-civil cooperation of the United Nations Office for the Coordination of Humanitarian Affairs (UN-OCHA) during an emergency was implemented. Particular attention was directed toward the control mechanisms used in the provision of emergency relief that was complicated by local military conflicts and acts of terrorism in Beslan. Stage I consisted of the collection and analysis of situational information, working out a plan of action, distribution of needs and deployment of human power and material resources with the interaction with the Ministry of the Russian Federation for Civil Defense, Emergencies and Elimination of Consequences of Natural Disasters (EMERCOM), and the Ministry of Internal Affairs, in response to the act of terrorism. Work in Stage I provided operational data from the situation, projection of the numbers of the injured, clarification of the general scheme for the medical-evacuation procedures, evacuation routes that could be used, the ambulance posts, and then management of prognosis of the injured. In addition, issues of medical triage and communication, the needs for evacuation transport, and informational support of the relief workers were identified.

During Stage II of the work, the conditions required for the admission of the injured to the republican hospital were prepared, and a pediatric field hospital was deployed that contained admission-triage, dressing, operational, and hospital-evacuation modules, as well as modules for support of the staff; all of the required processes for the "door-todoor" flows of injured/ill patients, and training of the responding medical personnel were prepared as well.

During Stage III, which continued for about four hours, the initial work with the injured was completed. "Door-todoor" triage was used to provide the required medical assistance and preparations were made for the urgent evacuation of many casualties to Vladikavkaz. A total of 120 beds for non-transportable and severely injured patients were reserved in the Central regional hospital. Every injured person was given about 1-7 minutes for triage and rendering immediate medical assistance. The main medical procedures were aimed at stopping internal and external hemor- rhages, at stopping asphyxia, normalizing breathing, and providing anti-shock measures. All severely injured children who couldn't be evacuated urgently underwent emergency surgery and resuscitation on-site.

During State IV, medical care was provided in the republican hospital of Vladikavkaz and the injured patients who required specialized medical care and follow-up treatment were prepared for air evacuation to medical institutions in Moscow and Rostov. During six days, there were 12 flights and 136 persons were evacuated-93 of them were children.

Keywords: air evacuation; children; disaster medicine; field hospital; military-civilian cooperation; response; Russia; terrorism Prehosp Disast Med 2005;20(2):s86

\section{Barents Rescue Exercise 2005-An Experience in Planning an International Arctic Search and Rescue Exercise Olaf Jacobsen; ${ }^{1}$ Raymond Teigen ${ }^{2}$ \\ 1. The Northern Norway Health Authorities, Norway \\ 2. The Northern Norway Health Authorities, Bodoe, Norway}

The Barents Rescue is a series of field training exercises organized and conducted by the countries of the Barents Euro-Arctic region. However, the exercise is open for international participation beyond the Barents region. It is an international field training exercise which focuses on challenges related to preparedness and emergency response in acute and serious crisis situations. It is an international challenge in weather-exposed and environmentally sensitive, remote areas in arctic waters. Particular emphasis is put on international, civil-military cooperation and interaction between the services from many countries that are mobilized in such situations. It gives practical, large-scale training for the whole trauma chain, including psychosocial work. The field training activities take place both at sea and on land.

The scenario evolves around crisis management after a terrorist attack on a large passenger vessel. The attack leaves a high number of casualties and a looming environmental threat at sea and along the coast. The scenario is developed in a manner that requires participation from many countries and numerous agencies and experts, such as rescue agencies, military, police, health, environmental agencies, and media experts.

As a part of the skill and competence building for trauma teams in the local rural hospital and key prehospital personnel in the Municipal Health Service, well-recognized practical trauma courses and lectures on triage, hypothermia, burns, and other selected themes open to all participants will be arranged.

The evaluation of the exercise, supported with existing knowledge about education, training, and lessons learned from similar exercises and real events, will contribute to the improvement of the international health response to disasters and other major events in the arctic region.

Keywords: Arctic; Barents Sea; cooperation; coordination; education; international

Prebosp Disast Med 2005;20(2):s86 